\title{
Evaluating the effects of information reliability on travellers' route choice
}

\author{
Gennaro Nicola Bifulco • Roberta Di Pace • \\ Francesco Viti
}

Received: 13 November 2012 / Accepted: 18 June 2013 / Published online: 6 July 2013

(C) The Author(s) 2013. This article is published with open access at SpringerLink.com

\begin{abstract}
Purpose This paper analyses travellers' behaviour with respect to route choice in a context where an Advanced Traveller Information System (ATIS) is in place. ATIS are important applications in the field of intelligent transportation systems (ITS). However, the practical impact of ATIS is still a matter for debate, and identification of expected route choice behaviour under ATIS is one of the main ways to assess their practical importance.

Methods Travellers' choices are frequently explored by means of stated preference (SP) approaches. In this paper we discuss some issues to be addressed when an SP survey is carried out, with particular reference to cases where a repeated choice approach is employed in the survey.

Results Our analysis concerns an application of the SP approach in a pilot study aimed at identifying the effects of ATIS accuracy on travellers' compliance with information. Conclusions This paper aims to make two major contributions. First of all, empirical analyses based on proper indicators and statistical tests are suggested in order to evaluate how the collected data have to be handled in order to eliminate transient route-choice observations. These are due to the warm-up phase inherently associated with the survey method adopted, dealing with repeated choices. Secondly, we analyse
\end{abstract}

\footnotetext{
G. N. Bifulco

Department of Transportation Engineering, University

of Naples "Federico II", Naples, Italy

e-mail: gennaro.bifulco@unina.it

R. Di Pace $(\bowtie)$

Department of Civil Engineering, University

of Salerno, Fisciano, Salerno, Italy

e-mail: rdipace@unisa.it

F. Viti

Faculty of Science, Technology and Communication,

University of Luxembourg, Luxembourg, Belgium

e-mail: francesco.viti@uni.lu
}

(stationary) route choice in order to assess the effects of information reliability (and the kind of information) on both route choice and compliance.

Keywords ATIS - Travel information - Stated preference . Travel simulator $\cdot$ Reliability $\cdot$ Route choice $\cdot$ Compliance . Uncertainty

\section{Introduction}

The aim of this paper is to ascertain whether and to what extent the way data are collected and used can affect the interpretation of experimental results obtained by stated preference (SP) surveys based on the use of travel simulators and applied through repeated choices. The final goal here is to assess the impacts of Advanced Traveller Information Systems (ATIS) on both travellers' compliance with information and route choice.

In recent decades ATIS applications have been a popular research topic for transportation analysts and many models have been proposed and discussed due to the widespread need to solve traffic oversaturation problems with ever-diminishing infrastructural investments. Moreover, from a commercial point of view, traffic information is also a valuable content for modern applications in the field of telecommunications. Indeed, some studies have shown that travellers exhibit a considerable willingness to pay for reliable advanced traffic information [27]. ATIS are intrinsically integrated with advanced communication platforms and devices, and aim to enhance (or integrate) the information level on network conditions that most travellers already have from their own traffic estimation process (experience). Information contents are gathered, elaborated and delivered by traffic control centres which are able to increase the reliability and effectiveness of (real-time) monitored traffic data.

Several researchers have studied the effect of information on traveller behaviour (see for instance [31]). In some cases 
analysis has explicitly focused on the dynamic process that results from the adjustment of travellers' choices over days, and in others the relationship between traffic-network stability and traveller behaviour is explored [33].

Travellers' behaviour is modelled with reference to adjustment processes, consisting of several mechanisms that determine the choices as these can be observed. Underlying these mechanisms the route choice/switching behaviour is considered [28-30, 34], where the risk perception associated with route choice plays a significant role $[2,6,20]$. Traveller's choice evolves over time in accordance with an updating process of the perceived travel times that underlie route choices [35]. This updating process is the so-called learning mechanism $[3,4,12,19]$. For a more extensive review of these approaches see also Viti et al. [40].

A major issue in the case of ATIS is to analyse the mechanism of compliance with the supplied information, defined as making choices consistent with the received information. This has been the subject of important studies, such as those by Van der Mede et al. [39], Mahmassani et al. [29], Chen et al. [18], Srinivasan and Mahmassani [36] and Chorus et al. [19]. In some cases the relationship between information compliance and information reliability has been explicitly investigated $[18,29]$, where the reliability is intended as the likelihood of the travel times the users actually experience on the network to be consistent with the supplied information.

Unlike other studies and/or other authors, we do not focus on the possible ways to model the day-to-day evolution of the travel choices and/or of the compliance, nor is any learning process addressed. We have a somewhat less ambitious goal, that is to identify choice behaviour in equilibrium conditions. This goal is pursued by means of a stated preference (SP) survey, where each respondent is subject to repeated choices in order to reveal his/her behaviour. In our analytical context it is crucial to identify when the respondents can be considered to be acquainted with the choice context and start to behave in a steady-state way, that is, when different responses can be observed just because of different stimuli and not because of a choice adaptation or updating process.

Indeed, in our experiment, which is based on a travel simulator and repeated choices, we identify a warm-up phase, related to the process carried out by the respondent in order to understand the experimental context. This is somewhat similar to the learning and updating mechanisms in route choices, described by day-to-day dynamic models of travellers' behaviour, but significant differences have to be taken into account. Our respondents undoubtedly spent some of the repeatedchoice trials learning about the network performance and reliability of the information system. However, in our experiment the network variables are constant: for any given respondent the network is the same in all trials (apart from randomness of actual travel times). This is different from the real world, where the learning and choice-updating mechanisms result in a dynamic evolution of the network as a whole, because of the congestion mechanism. The inconsistency between the respondents' learning and the network is not a matter in our experiment, since the analysis is disaggregated and the respondents do not actually experience congestion. In this context, the warm-up phase mainly aims at the quickest convergence to a stationary behaviour in route choice. Moreover, the dynamics of the warm-up process in our experiment is not the subject of our investigation. One of the tasks presented in this paper is to attain some evidence that the respondents become acquainted with the SP platform, assuming that the system we want to observe is in a steady-state condition. The realism of the steady-state hypothesis has often been criticised; however, many of the travel and transportation models are based on such a hypothesis $([15,17,19,20,26,27,41]$; etc.). Evidence of steady state is supported by three indicators, computed over the repeated choices of each respondent in order to check their stability. These analyses are preliminary to our identification of travellers' behaviour and are discussed in greater detail in Section 3.1.

As previously mentioned, the motivation for which it is worth studying compliance with information is strictly related to network effects. In some cases $[8,10]$, it has been proven that a high level of compliance is required in order for ATIS to be effective. However, the relationship between compliance and reliability of information (with reference to different kinds of information) has often been argued but not in all cases proven. According to widely used definitions, we state herein that the information is reliable when the estimates made by the ATIS are consistent with the travel times the travellers have actually experienced once at destination. This is determined by a combination of forecasting methods from the ATIS side, variability in travel times from the network side and travellers' experiences. The result is that compliance is not always expected to be high. This difficulty is exacerbated in the case of congested networks by the socalled anticipatory route guidance problem (ARG problem, e.g. $[16,24])$. In order to obtain reliable information, the suggestions, based on the estimates of the predicted state of traffic conditions, should consider travellers' reactions to the information itself. These reactions are however dependent on the actual compliance, and thus even in forecasting models that account for travellers' reactions $100 \%$ reliability is not expected to be met if compliance behaviour is not fully captured. These complex interactions defend our interest in compliance but are excluded from our experiment, since it is much more useful for studying the respondents' behaviour to treat reliability as an external parameter. In particular, reliability is the main design parameter of our experiment, since the effects of different reliability levels on compliance with information is one of the main interests in this work.

Another question arises in the real world (and also in our experiment), whether the compliance is actually observable 
and not only the concordance (see [7]). In fact, in our opinion, a traveller should be defined as compliant with ATIS when he/she chooses the suggested route because he/she trusts in the suggestion, whilst a traveller could be observed to be concordant also because he/she would have chosen the route regardless of the information advice. Therefore, concordance can be observed by the sole agreement between travellers' choices and information advice, while compliance is guaranteed when travellers' follow the advice irrespective of their current expectations. On the basis of previous considerations, the set of concordant travellers contains the set of compliant travellers. As a result, strictly speaking, all our analysis in this paper applies to concordance. However, having made this clarification, we will refer to the term compliance instead of concordance since it is accepted worldwide and widely used under the same meaning we intend for concordance.

In order to observe compliance and route choice behaviour under ATIS we used a web-based tool, such as those by Bogers et al. [14] and Lu et al. [28]. In our case the tool was the SP Platform, designed at the University of Naples [9], used to run experiments with a view to analysing travellers' behaviour in ATIS contexts [5]. The typical experimental procedure is such that respondents are invited to connect to a web page where they receive some basic information on the type of trip and on the travel alternatives available in the simulation. They are subsequently required to interact with the platform by choosing the route for their trip in a context when information is supplied. They are requested to make their repeated choices for several successive trials considering the suggestions of the simulated information system. Repeated trials for the same respondent serve two purposes: 1) to enable/allow the respondents to become familiar with both the network context and the ATIS performance (warmup phase, in the meaning it assumes in the context of our experimental work); 2) to observe several reactions of the same respondent with respect to different stimuli. The experiment carried out with the SP Platform is then interpreted under the basic assumption that a given number of trials is required to reach objective 1) while the following trials allow for the interpretation of the travellers' route choice behaviour in a stationary frame.

This paper is organised as follows: in Section 2 the SP Platform is briefly described and the experiment design and data collection are shown and discussed; in Section 3 analyses are carried out and results presented; in Section 4 conclusions in terms of major findings are discussed.

\section{The experiment}

In order to model travellers' behaviour, observation of real choices is needed. Generally this can be done by using two main approaches: that of stated preferences (SP) and revealed preferences (RP). In the first case travellers' responses are obtained by considering a hypothetical context of choice, whereas in the second case they are obtained by considering the choices actually made in a real context. The most widely adopted approach in the literature is that of stated preference [41]. Even if data acquired by RP [37] can be considered more realistic, the RP experiment is not suitable for investigating new choice contexts, new services, or systems with drastically new performance or characteristics. Moreover, RP surveys are more expensive than SP and the analyst has much less control in the experimental context. On the basis of the above considerations, the approach adopted in this research was that of SP, carried out by using the SP Platform [9], where the input variables considered in the design of the experiment are information reliability and the actual travel time variability of the network simulated by the platform.

\subsection{The SP platform}

The SP Platform (Fig. 1) is a web-based tool aimed at observing travellers' route choices in simulated Intelligent Transportation Systems (ITS) contexts. Travel simulators have been adopted in several cases in order to carry out SP experiments $[1,3,4,6,11,21,29]$.

The SP Platform is implemented in a highly flexible way and is virtually suitable for any kind of SP experiment related to route choices. The development of this tool has been strongly influenced by an existing one, the Travel Simulator Laboratory or TSL [22, 25], developed at the Delft University of Technology. We gained familiarity with the TSL during previous research and sought to replicate and improve its performance

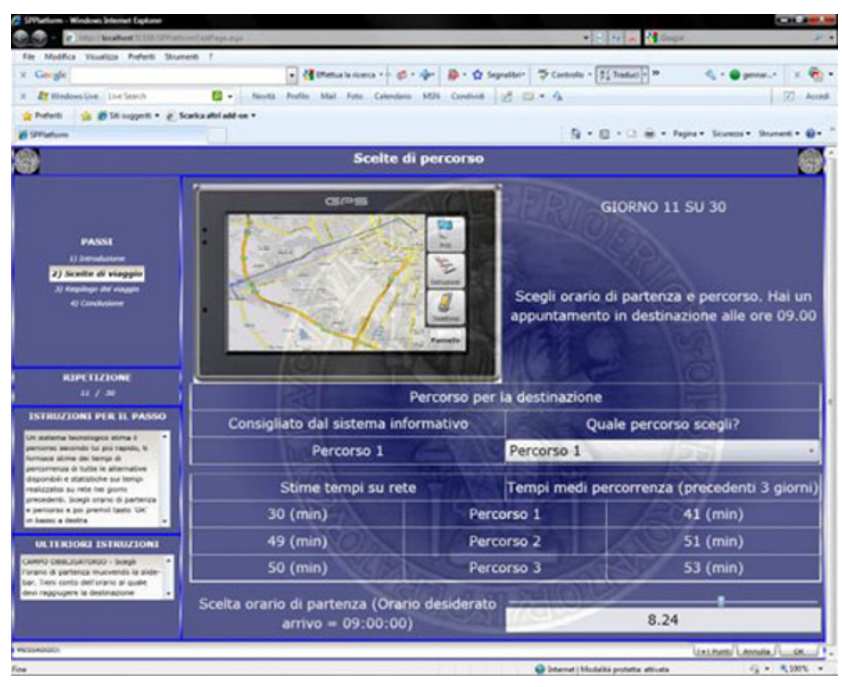

Fig. 1 Snapshot of the SP Platform at the information supply step (the experiment was in Italian) 
within the SP Platform. Most of the improvements concerned platform informatics, aimed at improving platform modularity, and are not of great interest to the topics dealt with in this paper.

\subsection{Design of the scenarios}

Here we discuss the experiment aimed at observing route choices in the presence of ATIS, implemented on the SP Platform, under different levels of information reliability and simulated travel time variability on the network. The information is simulated by means of a variable message sign (VMS) at a diversion node of the network (see Fig. 2).

The distribution of actual travel times on the network (as simulated by the platform) is one of the main input variables of the experiment. Three routes are available for the respondent, for each of which the information system applies an error. This simulates the estimation process of the ATIS with respect to the forecasting of actual travel times. The error made by the information system is the discrepancy between the actual travel times (as simulated by the platform) and the estimates made. These estimates are presented to the respondent at the VMS and the respondent is expected to take it into account in choosing a route, also in consideration of his/her own experiences and expectations of travel times on previous days. The three alternative routes are characterised differently in terms of average travel times.

The simulator also applies some predefined dispersions to these average travel times. According to these dispersions, in different trials the actual (simulated) travel times associated to a given route are different. On the basis of previous characteristics, the routes can be distinguished in terms of actual travel times and time reliability (variance of actual travel times). Route 1 is the fastest in terms of travel times (42.83 $\mathrm{min})$ but is not time-reliable in terms of standard deviation $(7.40 \mathrm{~min}$, with a coefficient of variation 0.17$)$. Route 2 is the longest in terms of travel times $(53.03 \mathrm{~min})$ and the one with the highest standard deviation (13.40 min, with a coefficient of variation of 0.25 ). Route 3 is slower than Route $1(52.10 \mathrm{~min})$ but is the most time-reliable in terms of standard deviation (1.55 min, with a coefficient of variation of 0.03 ). Note that Route 2 is dominated by Route 1 and 3 in terms of both average travel times and time-reliability. Two

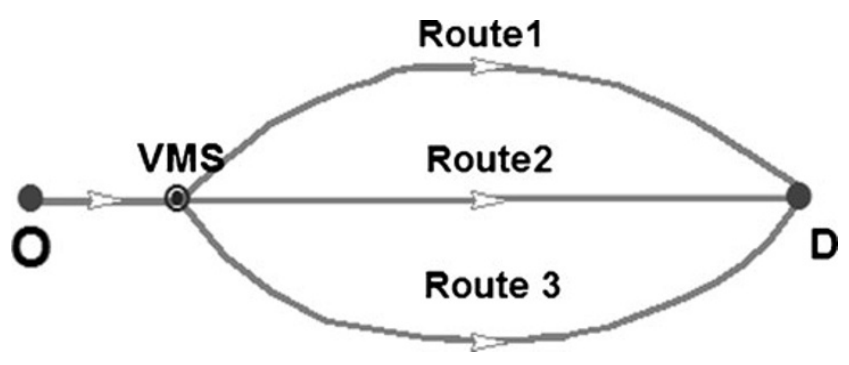

Fig. 2 Network description main kinds of route choice behaviours are considered to be plausible: either the respondent chooses the route perceived/ expected to be the fastest, or a very conservative behaviour is applied and the most time-reliable route is chosen. As a result it can be assumed that, if correctly perceived, Route 2 is the useless alternative, expected to be disregarded once travellers get acquainted with the choice context in the SP experiment.

In order to model the relationship between information reliability and compliance, three levels of reliability were considered. In the case of high and intermediate information reliability, the error of the ATIS-estimated travel times is drawn from a normal distribution with zero mean and with standard deviation proportional to each route's coefficient of variation $(\mathrm{CVj}$ for route $\mathrm{j})$. In the case of high reliability, the standard deviation is $0.25 * \mathrm{CVj}$; where the reliability level is intermediate, it is computed as $0.60^{*} \mathrm{CVj}$. For low reliability, a uniform random distribution was applied whereby the considered boundaries $(\mathrm{a}, \mathrm{b})$ are $85 \%$ of the minimum value among all simulated actual travel times and $115 \%$ of the maximum value among all simulated actual travel times (see Table 1).

In accordance with the classification widely used in the literature (e.g. [32]), the respondents are provided with different kinds of information. In the case of prescriptive information a suggestion for the fastest route is supplied. In the case of mixed information a combination of prescriptive and descriptive information is applied, where the descriptive part consists in supplying the (ATIS-estimated) travel times for all alternative routes. For mixed with augmented information, the augmentation consists in also supplying the averages of the actual travel times over the last three trials. It is worth noting that in all cases the ATIS estimates of travel times are employed to compute the ATIS-estimated fastest route, which is suggested to travellers. Thus the ATIS reliability perceived by the travellers depends on whether the suggested route actually proves to be the fastest. In our experiment, the number of times in which information is reliable in scenarios 1, 2 and 3 (high, intermediate and low reliability) is respectively $18 / 20,11 / 20$ and $6 / 20$. In practice, in the low-reliability scenario the suggestion is right a little less than one third of the times. In all cases respondents are also provided, at the end of the simulated trip, with feedback on the actual travel times of all routes (see Fig. 3).

\subsection{Data collection}

Respondents are requested to make their choices 30 consecutive times (trials). From trial 1 to trial 10 the ATIS is not simulated; it is considered to be in place starting from trial 11. Thus the experiment can be considered as divided into two parts: in the first part respondents' choices are influenced only by experience; in the second part also by the supplied information. Indeed, during the first part of the experiment the 
Table 1 Information error distribution/information distribution

\begin{tabular}{|c|c|c|c|c|}
\hline \multicolumn{2}{|l|}{ Levels of reliability } & \multirow{2}{*}{$\begin{array}{l}\text { Average } \\
0\end{array}$} & \multicolumn{2}{|c|}{$\begin{array}{l}\text { Standard deviation and } \\
\text { coefficient of variation }\end{array}$} \\
\hline \multirow[t]{6}{*}{ Error (Gauss distribution) } & High $(18 / 20)$ & & $0.25 \mathrm{CVj} x$ & 0.07821 \\
\hline & & & & 0.03491 \\
\hline & & & & 0.00757 \\
\hline & Intermediate $(11 / 20)$ & 0 & $0.60 \mathrm{CVj} x$ & 0.18769 \\
\hline & & & & 0.08378 \\
\hline & & & & 0.01817 \\
\hline \multirow[t]{3}{*}{ Travel time (Uniform distribution) } & Low $(6 / 20)$ & Route 1: 50.70 & $(b-a)$ & 14.55 \\
\hline & $a=0.85 *$ Min Actual $T T_{j}$ & Route 2: 54.08 & $\frac{\sqrt{12}}{\sqrt{12}}$ & 10.60 \\
\hline & $b=1.15 *$ Max Actual $T T_{j}$ & Route 3: 52.88 & & 5.99 \\
\hline
\end{tabular}

respondents are expected to become familiar with the network and with the pattern of travel times (and their dispersion and time reliability). Similarly, during the first trials of the second part, the respondents get familiar with the ATIS and its performance (e.g. information reliability).

On combining the three kinds of information (prescriptive, mixed and mixed with augmented information), with the three levels of reliability (high, intermediate, low), nine scenarios result. This can be considered as a pilot experiment, intended to gather evidence on how to manipulate the data obtained by the survey in order to avoid serious analytical distortions. For this reason, only ten respondents were randomly assigned to each scenario (90 respondents were involved in the experiment). However, the nature of the experiment has to be carefully considered, given that due to economic reasons it is not possible at the moment to select a fully representative sample.

The sample was composed as follows: $77.5 \%$ were male and $22.5 \%$ female; in terms of educational attainment $39 \%$ had a bachelor's degree or less, $40 \%$ had a master's degree, and $21 \%$ a $\mathrm{PhD}$; as regards age, $30 \%$ were between 23 and

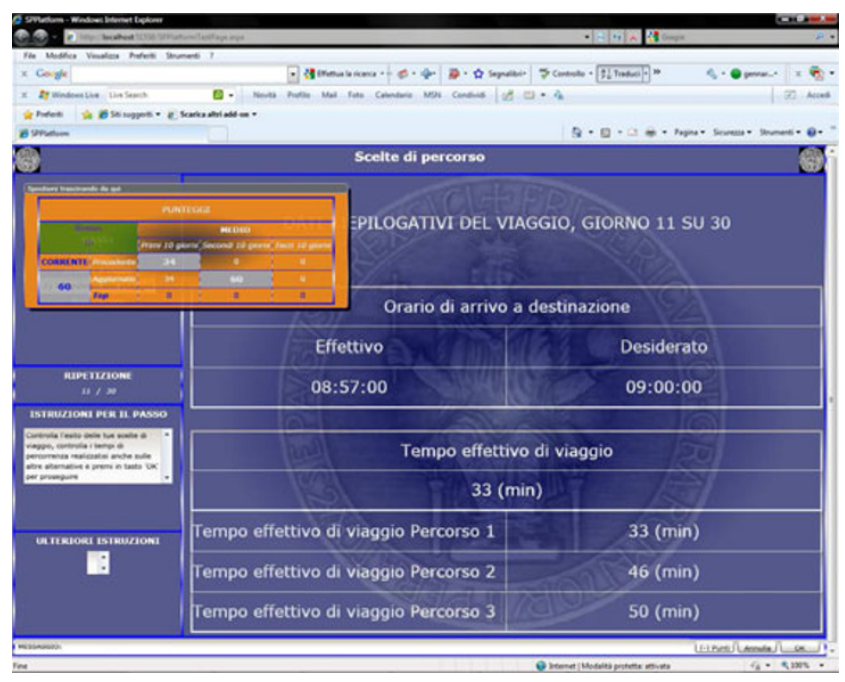

Fig. 3 Snapshot of the SP Platform at the feedback step (the experiment was in Italian)
28 years old, $20 \%$ between 29 and 35, and $30 \%$ between 36 and 70 years old; with reference to employment $29 \%$ of the sample were freelancers or non-university teachers, $51 \%$ students and $19 \%$ researchers or faculty members at university. The sample is clearly not representative of the population and respondents were recruited on a voluntary basis at the University of Naples. Strictly speaking, our results should be applied only to some segments of the population. For instance, the analyses reported below can be directly applied in the case of a population commuting to university. Otherwise, the main distortion of the results is that students and faculty members are used to arriving in time to attend classes; thus their behaviour is much more risk-adverse than risk-seeking. Since this could influence directly and indirectly both route-choice behaviour and compliance with information, the validity of the analyses in Section 3.2 should be revisited in the case of a different population.

For each respondent 30 repeated trials were observed. The motivation for the respondent was, at each trial, to reach the destination in time for a meeting (or to attend a class). The more punctual the respondents were able to be, the higher the assigned score was ( 50 points maximum). Moreover, a bonus of 10 points was assigned to each trial if the respondent was able to choose the fastest route (see [13]). The bonus sums up to the standard punctuality-related score. The rewards scheme was chosen by means of a pre-pilot study among ten friends of the SP platform developer (who is one of the authors of this paper). With this rewards scheme, most of the pre-pilot group were able to achieve almost the same score by acting in two different explicitly requested ways. The first was to consider the fastest-route bonus score as the main goal and the punctuality score as the secondary one, and vice versa for the second way. During the balancing of the rewards scheme only a context without ATIS was applied by the SP platform. Half of the pre-sample was asked to first act with the fastest-route goal and then in the more conservative way; vice versa for the other half of the pre-sample. Because of the balancing of the rewards scheme, we are confident that it does not discourage risk-adverse (preferring punctuality) or riskseeking (preferring the bonus) route choice behaviour. 


\section{Analysis of results}

With reference to the experimental context described in Section 2, in this paper we analyse: a) the number of trials required to make the respondents familiar with the network and the ATIS; b) the effect of different kinds of information and different levels of reliability on travellers' compliance and on route choices. In particular, we analyse whether in the first phase (without information) the travellers become familiar with the travel time distributions, and whether in the second phase (with information) they become familiar with ATIS reliability. We then explore the relationship between reliability on the one hand and compliance and route choices on the other.

\subsection{Warm-up phase}

In our experiment the respondents learn about the travel times every time they choose a route, at each trial of their repeatedchoice survey. With some similarities (and major differences, as already discussed) with the real world, in this warm-up period the respondents are aware of the highly limited knowledge about the route characteristics and regard their choices as taking a considerable degree of uncertainty into account. The more they experience the network, the more they become aware of the travel times, how much delay they may experience and so on. It is worth noting again that in our experiment the network performance is stationary and the differences of simulated actual travel times in successive trials only depend on random dispersion. Such a randomness is higher where unreliable travel times are simulated by the SP platform; however, in our experiment time reliability (unlike information reliability) is fixed for all simulated scenarios, albeit differently distributed across different routes.

In the presence of ATIS, travellers combine past experiences and ATIS estimates of the actual travel times in order to attain a higher degree of confidence in their expected travel costs. This is somehow similar to what happens in the real world [14]. In this context, they dedicate part of the warm-up phase to assessing the reliability of the information system.

As the number of trials needed for a respondent to become acquainted with the choice alternatives and characteristics is not evident, we chose to use two different metrics: the score, as assigned by the rewards structure of the SP Platform, as discussed in previous Section 2, and the percentage of respondents not choosing the useless route (route 2). We assume in this case that the respondents will not use the useless route once they have learnt that it is highly unlikely to be the best travel option either when a risk-seeking attitude is applied or when more conservative behaviour is adopted.

For the second part of the experiments (from Trial 11) another indicator is added, which is the percentage of compliant travellers. We expect this latter indicator to reach a stable value when the respondents have learnt about the reliability of the ATIS. All the previous indicators are analysed with reference to the different scenarios we tested in our experiment. In the second phase of the experiment, while the ATIS is in place and after acquaintance with the ATIS, we still use the score as an indicator for experiment fatigue; if the respondent is able to keep his/her score high, we assume that neither fatigue nor distraction played a role. It should also be considered that all the trials took place for twenty minutes on average, which is a fairly acceptable duration.

In order to ascertain how many trials the respondents needed to warm up, a non-parametric test (Kruskall Wallis) was used, with a significance level fixed at 0.05 . Being nonparametric, unlike the ANOVA test, the Kruskall Wallis test does not make assumptions on the distribution of the data.

As already stated, the tests were designed to assess the significant effect of the number of trials on:

1) arrival score, see Table 2 ;

2) tendency to avoid the useless route 2, see Table 3;

3) compliance with the suggested information (if any), see Table 4.

In all tables the reported values show the probability of the compared groups differing with respect to the evaluated performance only by chance. In practice, low values $(<0.05)$ allow us to state that the groups are (likely to be) significantly different (actually, non-equal).

The first test on the arrival score aims to show the significant effect of information. The reference step size is equal to five trials; in practice, we test whether there is an effect on arrival scores when respondents are evaluated for the first five trials, the second five or the third five.

In Table 2, a low number in comparing one data series with another (for instance, 0.001 for the overall comparison of all data series in the intermediate scenario) indicates that the variable is significantly different, that is the behaviour on the choice changes from one series to the other. Conversely, if the value is greater than 0.05 , it means that the behaviour with respect to route choice is confirmed between one series and another.

Results show that in the case of "no ATIS", the arrival scores during the first trials (from trials 1 to 5) are significantly different from the arrival scores from trials 6 to 10 ; in the case of high and intermediate levels of reliability, arrival scores during the first trials (from 11 to 15 ) are significantly

Table 2 Effect of trials on experiment score (kruskall-wallis test)

\begin{tabular}{lllll}
\hline Trials & $\begin{array}{l}\text { No } \\
\text { ATIS }\end{array}$ & $\begin{array}{l}\text { High } \\
\text { reliability }\end{array}$ & $\begin{array}{l}\text { Intermediate } \\
\text { reliability }\end{array}$ & $\begin{array}{l}\text { Low } \\
\text { reliability }\end{array}$ \\
\hline$[1 ; 5]$ vs $[6 ; 10]$ & $\mathbf{0 . 0 2 3}$ & & & \\
{$[11 ; 15]$ vs $[16 ; 20]$} & & $\mathbf{0 . 0 0 1}$ & $\mathbf{0 . 0 2 1}$ & $\mathbf{0 . 0 3 8}$ \\
{$[15 ; 20]$ vs $[21 ; 25]$} & & 0.263 & 0.339 & 0.562 \\
{$[21 ; 25]$ vs $[25 ; 30]$} & & 0.899 & 0.667 & 0.433 \\
\hline
\end{tabular}


Table 3 Effect of trials on tendency to avoid route2 (Kruskall-Wallis test)

\begin{tabular}{lllll}
\hline Trials & No ATIS & $\begin{array}{l}\text { High } \\
\text { reliability }\end{array}$ & $\begin{array}{l}\text { Intermediate } \\
\text { reliability }\end{array}$ & $\begin{array}{l}\text { Low } \\
\text { reliability }\end{array}$ \\
\hline$[1 ; 5]$ vs $[6 ; 10]$ & $\mathbf{0 . 0 0 5}$ & & & \\
{$[11 ; 15]$ vs $[16 ; 20]$} & & $\mathbf{0 . 0 0 0}$ & $\mathbf{0 . 0 0 7}$ & $\mathbf{0 . 0 2 4}$ \\
{$[15 ; 20]$ vs $[21 ; 25]$} & & 0.333 & 0.109 & 0.066 \\
{$[21 ; 25]$ vs $[25 ; 30]$} & & 1.000 & 0.327 & 0.065 \\
\hline
\end{tabular}

different from the arrival scores during trials from 16 to 20; the differences are not significant, for a given reliability level, if the arrival scores from 15 to 20 are compared to those from 21 to 25 and if the arrival scores from trials 21 to 25are compared to those from trials 25 to 30 .

This can be interpreted, with respect to the "No ATIS" stage of the experiment, as a significant difference (effect) probably because travellers had already learned during trials 6 to 10 , unlike during the first five trials. With respect to the ATIS stages, differences across trials can be interpreted as the effect of providing information (starting from trial 11) to the respondents' ability to arrive in time (or choose the fastest route, since both types of behaviours are rewarded). This effect does not significantly change from trial 21 to 30, probably because travellers had already learned during first informed trials. This applies to both the high and intermediate reliability levels, but it is less evident for the intermediate one, probably because the worse information reliability somewhat confuses the respondents. However, in the case of low reliability no significant effect of the information can be observed (from the first ten trials to the second ten, nor in the third ten). This may well be because respondents attained arrival scores that were too low almost in every case; in practice this can be interpreted as the information being too poor to have an effect.

In the second and third tests, the reference step size is equal to five trials for all the survey. With reference to the tendency to avoid route 2, as shown in Table 3 , in case of no ATIS (first ten trials), a significant difference is shown from trials $1-5$ to 6-10. This can be interpreted as follows: the respondents spend the first five trials understanding the main characteristics of the network (actually, the fact that route 2 is useless).

Moreover, in case of trials with information, a significant effect is shown on comparing trials 11 to 15 with trials 15 to 20 ( 0.000 at high reliability; 0.007 for intermediate reliability;

Table 4 Effect of trials on compliance (kruskall-wallis test)

\begin{tabular}{llll}
\hline Trials & $\begin{array}{l}\text { High } \\
\text { reliability }\end{array}$ & $\begin{array}{l}\text { Intermediate } \\
\text { reliability }\end{array}$ & $\begin{array}{l}\text { Low } \\
\text { reliability }\end{array}$ \\
\hline$[11 ; 15]$ vs $[16 ; 20]$ & $\mathbf{0 . 0 0 0}$ & $\mathbf{0 . 0 0 9}$ & 0.330 \\
{$[15 ; 20]$ vs $[21 ; 25]$} & 0.133 & $\mathbf{0 . 0 1 9}$ & 0.183 \\
{$[21 ; 25]$ vs $[25 ; 30]$} & 0.086 & 0.956 & 0.084 \\
\hline
\end{tabular}

0.024 for low reliability). A possible interpretation is that respondents behave in the first informed trials (trials 11 to 15) in a different way with respect to the useless route. Somehow, they have to re-adjust their behaviour (with respect to route 2) once the ATIS is in place and this happens during the first five informed trials. Subsequently, the respondents' behaviour with respect to route 2 seems to be the constant as trials run (they have gathered, say, that route 2 is useless also in the presence of the information system). This is much less evident for the lowreliability scenario, when the respondents' behaviour is somewhat disturbed by the unreliable information.

The same kind of test was carried out on compliance (see Table 4). In the case of high-reliability information, compliance in the first five informed trials (11 to 15$)$ differs significantly from trials 16 to 20 . This does not apply on comparing trials 15 to 20 with 21 to 25 , nor on comparing trials 20 to 25 with the 26 to 30 . This means that compliance is in some way established after the first five informed trials (from trial 15). A similar behaviour applies at the intermediate-accuracy information level. However, in this case the difference between the second and third group of trials is significant, meaning that more trials (up to trial 20) are required by respondents in order to stabilise their compliance with the supplied information. In the case of the low-accuracy level, the differences are always significant; this is consistent with the interpretation that respondent compliance is somehow unstable: the respondents are always uncertain whether or not to be compliant (or, at least, a clear-cut attitude cannot be identified).

On the basis of the results obtained, we can conclude that in our experiment:

- the first five trials (without information) seem to be enough for respondents to understand how the network performs (at least the main route characteristics);

- when information is introduced into the experiment the respondents change their route choice behaviour and the new behaviour needs more or fewer trials to be clearly identifiable depending on the reliability of the supplied information;

- stronger evidences (such as the uselessness of route 2) are easier to recover in the respondents' awareness; but, in the case of scarce information reliability, more trials are still needed in order to recover respondent awareness;

Table 5 Aggregate choices at different reliability levels (all kinds of info)

\begin{tabular}{llll}
\hline & \multicolumn{2}{l}{ Route Share (\%) } & Compliance (\%) \\
\cline { 2 - 3 } Level of reliability & \multicolumn{2}{l}{ Faster route } & Most reliable route \\
\hline High & 71.76 & 22.50 & 85.00 \\
Intermediate & 61.31 & 20.66 & 66.97 \\
Low & 45.33 & 27.00 & 64.17 \\
\hline
\end{tabular}


- compliance with information is, as expected, more sensitive to information reliability: in the case of high reliability it is quickly established; under low reliability it is very hard to establish and tends to be highly variable with trials; in the case of intermediate reliability some more trials than in the high-reliability case are needed by the analyst in order to be confident that stable compliance is observed.

3.2 The effect of reliability: compliance with information and route choices

The previous section (3.1) showed that the analyst is likely to observe a somewhat stable behaviour of the respondents after they have familiarised themselves with the experiment. The first 20 trials (including those without information) are enough to allow for this process in the case of high and intermediate reliability, and this happens for any of the indicators considered. In the case of low reliability, compliance is not easy to be identified over trials throughout our experiment. On the basis of these considerations, the first 20 trials were removed from the analysis aiming to understand travellers' route choice behaviour. Results for the low-reliability level should be treated with less confidence than the other two levels of reliability.

Table 5 shows that, on the basis of aggregate percentages, as expected, compliance is strictly related to information reliability (see also [23]). In particular, compliance decreases in accordance with the reliability level. This effect is more evident from the most reliable to the intermediate level of reliability than from the intermediate to the lowest level. In the case of high reliability, a high tendency to choose the fastest route can also be noted (also because the suggested route tends to be the fastest one in the case of reliable ATIS), as noted also in Ben-Elia et al. [5]. This tendency also decreases according to the reliability. In the case of the least reliable information scenario, a higher tendency to choose the most time-reliable route can be observed.

These results can also be interpreted in terms of risk-adverse or risk-seeking attitudes by travellers. For instance, Avineri and Prashker [4] analysed how people react to information when they have to make a decision in uncertain conditions. Using an SP survey they studied the influence of providing static and dynamic information on a two-route network. It was found that respondents tended to choose a faster but time-unreliable route less often when no information regarding routes was given, while in the case of information, the preference toward the faster route increased. Our result is consistent with this, as well as with other similar findings in literature (e.g. [40]).

A more disaggregated analysis allows, for our experiment, some differences to be identified in the case of different information types (see Table 6).

The results show that in all cases at the highest levels of reliability respondents are highly compliant (they tend to choose route 1 , which is most of the time both the fastest
Table 6 Aggregate choices and compliance (without warm-up data)

\begin{tabular}{lllll}
\hline Information & \multicolumn{2}{l}{ Route share (\%) } & $\begin{array}{l}\text { Compliance } \\
(\%)\end{array}$ \\
\cline { 2 - 4 } & $\begin{array}{l}\text { Lev of } \\
\text { reliability }\end{array}$ & $\begin{array}{l}\text { Faster } \\
\text { route }\end{array}$ & $\begin{array}{l}\text { Most rel. } \\
\text { route }\end{array}$ \\
\hline Prescriptive & High & 76.07 & 22.50 & 94.47 \\
& Intermediate & 70.00 & 14.23 & 74.61 \\
& Low & 54.54 & 21.36 & 66.81 \\
Mixed & High & 69.00 & 21.50 & 82.00 \\
& Intermediate & 63.00 & 19.00 & 77.33 \\
\multirow{3}{*}{ Mixed } & Low & 37.27 & 33.45 & 62.72 \\
information & High & 68.50 & 23.50 & 74.50 \\
& Intermediate & 47.50 & 31.50 & 41.50 \\
& Low & 43.75 & 23.13 & 61.00 \\
\hline
\end{tabular}

route and that suggested by the system). In the case of prescriptive information and mixed information, the travellers' tendency to choose the most time-reliable route (route 3) increases with information unreliability, and the share of the preferences for the useless route also increases. This confirms that when the reliability of the information decreases:

1. travellers get more confused and are less able to identify the fastest and the most time-reliable routes;

2. also due to this confusion, travellers still maintain some degree of compliance (optimistic anchoring, [38]) and they follow the suggestions even when the useless route is suggested;

3. the previous behaviour occurs more frequently as the ATIS becomes less reliable and supplies useless information more frequently.

In the case of mixed information with augmentation and low (but also intermediate, to some extent) levels of information reliability the respondents seem to be very confused by the presence of ATIS. Unexpectedly, compliance attains a value of more than $60 \%$ even if the information is unreliable; moreover, the share of the useless route is extremely high (33\%). In practice what we are observing is false compliance.

The observed pattern is consistent with the interpretation that the respondents choose almost totally at random and, provided that the information system also suggests the route in an almost random way, only by chance does the route chosen by the respondent frequently coincide with the suggested route.

\section{Conclusions}

The focus of this paper was on travellers' route choices under ATIS. In particular, an experiment was carried out by considering different inaccuracy levels and different kinds of information: 
Table 7 Aggregate choices and compliance (only warm-up data)

\begin{tabular}{lllll}
\hline Information & \multicolumn{2}{l}{ Route share (\%) } & $\begin{array}{l}\text { Compliance } \\
(\%)\end{array}$ \\
\cline { 2 - 4 } & $\begin{array}{l}\text { Level of } \\
\text { reliability }\end{array}$ & $\begin{array}{l}\text { Faster } \\
\text { route }\end{array}$ & $\begin{array}{l}\text { Most rel. } \\
\text { route }\end{array}$ \\
\hline Prescriptive & High & 55.71 & 24.28 & 95.00 \\
& Intermediate & 53.63 & 12.72 & 76.36 \\
& Low & 45.00 & 24.16 & 60.83 \\
Mixed & High & 38.00 & 26.00 & 79.00 \\
& Intermediate & 40.00 & 30.00 & 82.14 \\
\multirow{4}{*}{ Mixed } & Low & 48.00 & 30.00 & 73.00 \\
information + & High & 48.00 & 13.00 & 68.00 \\
& Intermediate & 37.00 & 34.00 & 59.00 \\
& Low & 45.56 & 21.11 & 62.22 \\
\hline
\end{tabular}

prescriptive; mixed; mixed with augmented information. On the basis of the data collected two different kinds of analyses were made: the first studied the warm-up phase of the experiment in order to determine from which trial of our repeated choice experiment suitable data for our route choice analysis can first be extracted. This analysis was based on the use of several warmup indicators and statistical tests. The empirical approach adopted is suggested for similar kinds of studies. Once the warm-up phase is properly identified, the second analysis aimed to assess the effect of information (with reference to different kinds of information and its reliability) on travellers' route choice behaviour. Non-parametric statistical tests showed that the respondents can be considered to have concluded the warm-up phase from trial 20 onwards.

If the warm-up trials are not removed, the risk of interpreting the experiment wrongly is high, as is the risk of being unable to identify any interpretable pattern in the results. This is confirmed by Tables 7 and 8 below, where route choice behaviour in the warm-up phase is analysed and the difference between the warmup and the non-warm-up data is tested by means of a nonparametric statistical test. Table 7 shows that route choice behaviour differs greatly from that in Table 6 , as well as being inconsistent with expectations. In particular, the share of the fastest route is quite low for any level of information accuracy, the share of the most time-reliable route is similar to the case of the nonwarm-up data (see Table 6) and the share of the useless route is (computed by difference) unreasonably high. This pattern shows the extent of the respondents' confused behaviour in the warmup phase. By looking at Tables 7 and 6 it seems that the difference in compliance in the warm-up and non-warm-up phase is much less evident. However, the statistical test (Table 8) with reference to compliance exhibits a value less than 0.05 , this suggests that the observed patterns can be considered to be different. The difference between the warm-up and nonwarm-up phases is confirmed by almost all statistics in Table 8, even if this is less evident in the case of low information accuracy where uncertainty prevails.
Table 8 Testing the difference: warm-up data vs. non-warm-up data (Kruskall-Wallis test)

\begin{tabular}{lllll}
\hline Information & \multicolumn{2}{l}{ Route share (\%) } & $\begin{array}{l}\text { Compliance } \\
(\%)\end{array}$ \\
\cline { 2 - 4 } & $\begin{array}{l}\text { Level of } \\
\text { reliability }\end{array}$ & $\begin{array}{l}\text { Faster } \\
\text { route }\end{array}$ & $\begin{array}{l}\text { Most rel. } \\
\text { route }\end{array}$ \\
\hline Prescriptive & High & 0.000 & 0.063 & 0.000 \\
& Intermediate & 0.001 & 0.035 & 0.000 \\
& Low & 0.029 & 0.458 & 0.000 \\
Mixed & High & 0.000 & 0.199 & 0.000 \\
& Intermediate & 0.000 & 0.070 & 0.000 \\
Mixed & Low & 0.005 & 0.088 & 0.000 \\
information + & High & 0.003 & 0.028 & 0.035 \\
& Intermediate & 0.007 & 0.003 & 0.003 \\
& Low & 0.563 & 0.656 & 0.000 \\
\hline
\end{tabular}

Excluding the warm-up phase from the analysis enabled the effect of different information reliability levels and different kinds of information to be better assessed. This was carried out by considering some aggregate indicators on route share and compliance. With reference to information reliability (without considering different kinds of information), our results show a significant effect on travellers' compliance (e.g. compliance decreases as unreliability increases). Indeed, information reliability seems to directly influence the route choices made by the respondents. At a higher level of reliability travellers mainly choose the fastest route (which is, also in this case, the most suggested route). However, when information reliability decreases, the choice context is perceived as being more uncertain and hence respondents tend to choose the most time-reliable route, or even the useless route. In these cases the respondents also comply less with the information received.

Of course, our results should be viewed as preliminary because the data considered were collected in the context of a pilot experiment. In future works, with a due increase in research funding, we intend to increase the number of respondents per scenario (and most importantly the assortment of the respondents, currently mainly recruited in an academic milieu). This should allow consolidation of the results presented and discussed herein.

It is worth noting that our results have important implications in terms of route choice modelling. Indeed, in order to calibrate a route choice model, information reliability needs to be incorporated as an explanatory variable.

Acknowledgments The authors are grateful to Prof. Giulio Erberto Cantarella for his suggestions and for the time patiently spent on interesting conversations about the topic discussed herein.

Open Access This article is distributed under the terms of the Creative Commons Attribution License which permits any use, distribution, and reproduction in any medium, provided the original author(s) and the source are credited. 


\section{References}

1. Adler JL, McNally MG, Recker W (1993) Interactive simulation for modelling dynamic driver behaviour in response to ATIS. http:// www.its.uci.Edu

2. Avineri E, Prashker JN (2003) Sensitivity to uncertainty: the need for a paradigm shift. Transp Res Rec 1854:90-98

3. Avineri E, Prashker JN (2005) Sensitivity to travel time variability: travelers' learning perspective. Transp Res C 13(2):157-183

4. Avineri E, Prashker JN (2006) The impact of travel time information on travelers' learning under uncertainty. Transportation 33:393-408

5. Ben-Elia E, Di Pace R, Bifulco GN, Shiftan Y (2013) The impact of travel information's accuracy on route-choice. Transp Res C 26:146-159

6. Ben-Elia E, Erev I, Shiftan Y (2008) The combined effect of information and experience on drivers' route-choice behavior. Transportation 35:165-177

7. Bifulco GN, Cantarella GE, de Luca S, Di Pace R (2011) Analysing and modelling the effects of information accuracy on travellers' behaviour. Proceedings of the $14^{\text {th }}$ IEEE ITSC Intelligent Transportation Systems Conference. doi:10.1109/ITSC.2011.6082961. doi:10.1109/ ITSC.2011.6082961\#blank, pp 2098-2105

8. Bifulco GN, Cantarella GE, Simonelli F (2013) Design of signal setting and advanced traveller information systems, accepted for publication. J Intell Transp Syst Technol Plan Oper. doi:10.1080/ 15472450.2013.802156

9. Bifulco GN, Di Pace R, Simonelli F (2009) A simulation platform for the analysis of travel choices in ATIS context through Stated Preferences experiments. EWGT Conference-Padua, Italy

10. Bifulco GN, Simonelli F, Di Pace R (2007) Endogenous driver compliance and network performances under ATIS. Proceedings of the IEEE ITSC Intelligent Transportation Systems Conference. Seattle, WA, USA, Sept. 30-Oct. 3. doi:10.1109/ITSC.2007.4357722. doi:10.1109/ITSC.2007.4357722\#blank

11. Bogers EAI (2009) Traffic information and learning in day to day route choice. PhD Thesis Dissertation TRAIL Research School

12. Bogers E, Bierlaire M, Hoogendoorn S (2007) Modeling learning in route choice. Transp Res Rec 2014:1-8

13. Bogers EAI, Hoogendoorn SP (2004) The impact of various uncertain information schemes on route choice In Proceedings of the ETC. Association for European Transport, Strasbourg, pp 1-19

14. Bogers EAI, Viti F, Hoogendoorn SP (2004) Joint modeling of ATIS, habit and learning impacts on route choice by laboratory simulator experiments. Transp Res Rec 1926:189-197

15. Bonsall PW, Parry T (1990) A computer simulation game to determine drivers' reactions to route guidance advice. Proc. $18^{\text {th }}$ Summer annual meeting of PTRC, London, pp 113-124

16. Bottom J, Kachani S, Perakis G (2007) The anticipatory route guidance problem: formulations, analysis and computational results. Proceedings for TRISTAN VI. http://www.utrc2.org/research/assets/ 99/RouteGuidance-Article1.pdf

17. Chang HL, Chen PC (2009) The impact of uncertain travel information on drivers' route choice behaviour. Transp Res B

18. Chen PS-T, Srinivasan KK, Mahmassani HS (1998) Effect of information quality on compliance behavior of commuters under real-time traffic information. Transp Res Rec 1676:53-60

19. Chorus CG, Arentze TA, Timmermans HJP (2009) Traveler compliance with advice: a Bayesian utilitarian perspective. Transp Res E 45:486-500

20. Chorus CG, Dellaert BGC (2012) Travel choice inertia: the joint role of risk aversion and learning. J Transp Econ Policy 46(1):139-155

21. Chorus CG, Molin EJE, Arentze TA, Hoogendoorn SP, Timmermans HJP, van Wee GP (2007) Validation of a multimodal travel simulator with travel information provision. Transp Res C 15(3):191-207
22. Chorus CG, Molin EJE, Arentze TA, Timmermans HJP, van Wee GP (2007) Travelers' need for information in traffic and transit: results from a web-survey. J Intell Transp Syst 11(2):1-11

23. Di Pace R (2008) Analytical tools for ATIS (Strumenti Analitici per Applicazioni ATIS). PhD Thesis, Università degli Studi di Napoli "Federico II"-Facoltà di Ingegneria

24. Dong J, Mahmassani Hani S, Lu C-C (2007) How reliable is this route?: predictive travel time and reliability for anticipatory traveler information systems. Transp Res Record: J Transp Res Board, pp $117-125$

25. Hoogendoorn SP. TSL homepage. Delft University of Technology, http://www.tsl.tudelft.nl/. Accessed July 2004

26. Khattak AJ, Scofer JL, Koppelman FS (1993) Commuters' en-route diversion and return decisions: analysis and implications for advanced traveller information systems. Transp Res A 27A(2):101-111

27. Khattak AJ, Yim Y, Stalker PL (2003) Willingness to pay for travel information. Transp Res C 11:137-159

28. Lu X, Gao S, Ben-Elia E (2011) Information impacts on route choice and learning behavior in a congested network experimental approach transportation research record: Journal of Research Record No. 2243. Transportation Research Board of the National Academies, Washington, DC, pp 89-98

29. Mahmassani HS, Jou RC (1998) Transferring insights into commuter behaviour dynamics from laboratory experiments to field surveys. Transp Res A Policy Pract 34(4):243-260

30. Mahmassani HS, Liu Y-H (1999) Dynamics of commuting decisions behavior under advanced traveler information systems. Transp Res C 7(2):91-107

31. Polydoropoulou A, Ben-Akiva M (1998) The effect of advanced traveller information systems (ATIS) on travellers' behaviour. In: Emmerink RHM, Nijkamp P (eds) Behavioural and network impacts of driver information systems. Ashgate, Aldershot

32. Schofer JL, Khattak A, Koppelman FS (1993) Behavioral issues in the design and evaluation of advanced traveler information systems. Transp Res C 1(2):107-117

33. Srinivasan KK, Guo Z (2007) Day-to-day evolution of network flows under route-choice dynamics in commuter decisions transportation research record. J Transp Res Board 198-208

34. Srinivasan KK, Mahmassani HS (1999) Role of congestion and information in tripmakers' dynamic decision processes: an experimental investigation. Transp Res Rec 1676:43-52

35. Srinivasan KK, Mahmassani HS (2002) Dynamic decision and adjustment processes in commuter behavior under real-time information Technical Research Report SWUTC/02/167204-1, Center for Transportation Research. University of Texas at Austin, February

36. Srinivasan KK, Mahmassani HS (2007) Modeling inertia and compliance mechanisms in route choice behavior under real-time information. Transp Res Record J Transp Res Board 45-53

37. Tsirimpa A, Polydoropoulou A, Antoniou C (2009) Development of latent variable model to capture the impact of risk aversion on travelers' switching behavior. J Choice Model 3(1):127-148

38. Tversky A, Kahneman D (1974) Judgment under uncertainty: heuristics and biases. Science 185:1124-1130

39. Van der Mede HJ, Van Berkum EC (1996) Modelling car drivers' route choice information environments, Delft University of Technology, Transportation Planning and Traffic Engineering Section Rudimental Contributions

40. Viti F, Bogers EAI, Hoogendoorn SP (2005) Day-to-day learning under uncertainty and with information provision: model and data analysis. Presented at the 16th International Symposium of Transport and Traffic Theory (ISTTT), University of Maryland

41. Wardman M, Bonsall PW, Shires JD (1996) Driver response to variable message signs: a stated preference investigation. Trans Res C 5(6):389-405 